\title{
Discrete Family of Dissipative Soliton Pairs in Mode-Locked Fiber Lasers
}

\author{
Aleksandr Zavyalov * Rumen Iliew, Oleg Egorov, and Falk Lederer \\ Institute of Condensed Matter Theory and Solid State Optics, \\ Friedrich-Schiller-Universität Jena, Max-Wien-Platz 1, 07743 Jena, Germany
}

(Dated: November 6, 2018)

\begin{abstract}
We numerically investigate the formation of soliton pairs (bound states) in mode-locked fiber ring lasers. In the distributed model (complex cubic-quintic Ginzburg-Landau equation) we observe a discrete family of soliton pairs with equidistantly increasing peak separation. This family was identified by two alternative numerical schemes and the bound state instability was disclosed by a linear stability analysis. Moreover, similar families of unstable bound state solutions have been found in a more realistic lumped laser model with an idealized saturable absorber (instantaneous response). We show that a stabilization of these bound states can be achieved when the finite relaxation time of the saturable absorber is taken into account. The domain of stability can be controlled by varying this relaxation time.
\end{abstract}

PACS numbers: 42.55.Wd, 42.65.Tg, 42.65.Sf, 42.79.Sz, 42.81.Uv

Keywords: fiber lasers, nonlinear optics, optical solitons, communication systems, bound states

\section{INTRODUCTION}

Since the experimental observation of stable solitary pulses (SPs) in optical fibers [1] their interactions attracted a great deal of interest. In the early stages the propagation of light pulses was described using the nonlinear Schrödinger equation (NLSE). Relying on a perturbation approach it was shown that SP interactions depend on the relative phase between them: in-phase solitons attract each other, while out-of-phase solitons repel [2, 3, 4, 5], and for an arbitrary phase the interaction appears more involved [5].

For a more realistic description of real systems different perturbations were added to the NLSE, accounting for losses [6], intrapulse Raman scattering [7, 8, 9], third-order dispersion [7, 8] or spectral filtering. For the description of a complete communication line with a periodic arrangement of signal regeneration (semiconductor amplifier, saturable absorber) and propagation in the fiber (effects as nonlinearity and absorption) a distributed description with the so-called complex cubicquintic Ginzburg-Landau equation (CQGLE) was established [11]. Another optical system of primary importance, which is commonly modeled by this equation, is the mode-locked fiber ring laser, because it essentially consists of the same optical components in a closed loop [12]. Despite its success in the description of mode-locked lasers, the assumptions used to derive the CQGLE are not always fulfilled in reality.

In this contribution we investigate the formation of bound states in fiber lasers exploiting the CQGLE and a more realistic lumped model. From exact numerical simulations of the CQGLE we obtain a discrete family of BS stationary solutions with different (equidistant) peak separation. Moreover, we show that a lumped laser model

*Electronic address: aleksandr.zavyalov@uni-jena.de which accounts additionally for finite temporal effects of the absorber can have profound consequences for the BS stability.

The CQGLE can be considered as a perturbed NLSE where the complex coefficients of dispersion and nonlinearity account for spectral filtering and saturation, respectively, and a linear loss/gain is added. For certain parameter sets this equation was shown to support solitary wave solutions, called dissipative solitons, as well as bound states (BS) of pairs of SPs [13, 14, 15, 16, 17, 18, 19, 20, 21]. Careful numerical investigations [16] have demonstrated BS formation from a pair of initially resting SPs. Depending on the dispersion regime and the initial phase difference the BS profile can vary. Furthermore it was shown [17, 18] that out-of-phase and in-phase SPs can form unstable stationary BS solutions, because they represent saddle points in the phase plane. In the framework of the CQGLE stable BSs were discovered by Akhmediev et al. 21] as stable two-soliton solutions with a $\pi / 2$ phase difference of the peaks. They were observed for anomalous dispersion only.

In an early work 14 it was analytically shown that these dissipative equations exhibit a discrete family of two-soliton solutions with different (equidistant) peak separation. This analysis was based on both a perturbation approach for the NLS equation with small pumping and dissipative terms and the CQGLE with weak anomalous dispersion. To the best of our knowledge, however, such discrete families of BSs have not yet been found numerically or experimentally.

In the first section of this paper we briefly present the equations used for modeling the fiber ring laser for different approximations. We depart from the more general lumped description, where all individual elements in the cavity are modeled by separate equations and the pulse is sequentially propagated through these elements in the ring cavity. Then, we derive the CQGLE from the lumped model by averaging over the full cavity. We obtain definite relations between the coefficients of both 
models which is essential for the comparison with experiments.

The second section is devoted to the discrete family of stationary BS solutions derived from the CQGLE. In the numerical simulations we observe a discrete family of stationary two-soliton solutions with different peak-to-peak separation which we identify as different BS levels. These results are obtained by means of two numerical schemes, viz., the solution of either the evolution or the stationary problem. Based on the results of the stationary analysis, a linear stability analysis is carried out where the instability growth rates perfectly coincide with the results of the propagation model.

For a more realistic description we take advantage of the lumped laser model in the third part, where the individual elements of the cavity are treated separately, and show that the different BS levels can be stabilized by accounting for a noninstantaneous, but very fast, response of the saturable absorber (SA), whereas for an instantaneous absorber response the BSs remain unstable. In the latter case the SA is described by the commonly used instantaneous response approximation (ideal SA).

Interestingly, by varying the SA response time and saturation level the BS solutions can be forced to change from stable to unstable behavior. Moreover, all these stable two-pulse solutions exhibit a multilevel nature and are located on a certain side of the phase plane depending on the level.

In what follows we are restricting ourselves to the normal dispersion regime because this is the realm of the so-called all-normal-dispersion lasers, which attract a considerable deal of interest presently. Compared to other fiber lasers they have a simpler setup and allow for achieving higher pulse energies. Energies as high as $256 \mathrm{~nJ}$ per pulse were achieved in a large-mode-area photonic crystal fiber laser [22], and more than $20 \mathrm{~nJ}$ for a usual single-mode fiber laser [23]. Pulses generated in all-normal-dispersion lasers depend nontrivially on the interplay of spectral filtering and self-phase-modulation [24].

\section{MODELS}

For the numerical modeling we use a simple scheme of a ring fiber laser, which consists of a doped fiber, a saturable absorber and an output coupler. This laser model allows for including the dominant effects into the simulations and is still close to reality. In the lumped model the propagation through each element is treated separately.

The propagation along the doped fiber is described by the modified nonlinear Schrödinger equation which is given by [7, 25, 26] (when the carrier optical frequency equals the dopant's atomic resonance frequency)

$$
\begin{aligned}
\frac{\partial U(z, t)}{\partial z}+ & \frac{i}{2}\left(\beta_{2}+i g(z) T_{1}^{2}\right) \frac{\partial^{2} U(z, t)}{\partial^{2} t} \\
& =\frac{g(z) U(z, t)}{2}+i \gamma|U(z, t)|^{2} U(z, t)
\end{aligned}
$$

where $U(z, t)$ is the envelope of the pulse, $z$ is the propagation coordinate, $t$ is the retarded time, $\beta_{2}$ is the second-order dispersion (GVD) coefficient, and $\gamma$ represents the fiber nonlinearity. $g(z)$ is the saturable gain of the doped fiber and $T_{1}$ is the dipole relaxation time (inverse linewidth of the parabolic gain). Assuming that the conditions are close to stationarity, the gain can be approximated by [27]

$$
g(z)=\frac{g_{0}}{1+\int_{\text {pulse }}|U(z, t)|^{2} d t / E_{\text {sat }}^{\mathrm{Gain}}}
$$

where $g_{0}$ is the small-signal gain, which is defined by the pumping level, and $E_{\text {sat }}^{\text {Gain }}$ is the saturation energy.

To describe the time-dependent semiconductor SA response we use the Agrawal/Olsson model [28], which we term noninstantaneous $\mathrm{SA}$ response and is given by

$$
\begin{aligned}
& \frac{\partial U(z, t)}{\partial z}=-\frac{1}{2} \delta(z, t) U(z, t) \\
& \frac{\partial \delta(z, t)}{\partial t}=\frac{\delta_{0}-\delta(z, t)}{T_{\text {relax }}}-\frac{\delta(z, t)|U(z, t)|^{2}}{E_{\mathrm{sat}}^{\mathrm{SA}}}
\end{aligned}
$$

where $\delta(z, t)$ is the loss introduced by the absorber, $\delta_{0}$ is the small-signal loss, $T_{\text {relax }}$ is the recovery time and $E_{\text {sat }}^{\mathrm{SA}}$ is the saturation energy. For $T_{\text {relax }}<T_{\text {pulse }}$ and taking into account that standard absorbers are thin, we obtain the well-known transmission equation for the SA in the instantaneous response approximation (ideal SA) from system (3)

$$
U_{\text {out }}(t)=U_{\text {in }}(t) \exp \left(-\frac{1}{2} \frac{\delta_{0} \Delta z}{1+\left|U_{\mathrm{in}}(t)\right|^{2} / P_{\mathrm{sat}}}\right),
$$

where $\Delta z$ is the length of the SA, $\delta_{0} \Delta z$ defines the modulation depth of the absorber and the saturation power is defined as $P_{\text {sat }}=E_{\text {sat }}^{\mathrm{SA}} / T_{\text {relax }}$.

This lumped model describes the experimental setup appropriately but it is too involved for analytical studies, including the linear stability analysis. In order to obtain a single equation with constant coefficients the periodic system is approximated by averaging over the full cavity length using the guiding-center soliton technique [29, 30], which relies on the field decomposition into a product containing a periodic part and an average amplitude. This model is justified if physically relevant changes appear upon several round-trips. For the sake of simplicity of the resulting equation the instantaneous response approximation for the SA (4) is used and the saturation of the fiber gain is neglected, i.e. we simplify Eq. 
(2) to $g(z) \approx g_{0}$ in the limit $\int_{\text {pulse }}|U(z, t)|^{2} d t \ll E_{\text {sat }}^{\text {Gain }}$.

Eventually we obtain a single evolution equation, which is a modified complex Ginzburg-Landau equation (MGLE) given by

$$
\begin{aligned}
i \frac{\partial V}{\partial Z}+\frac{D}{2} & \frac{\partial^{2} V}{\partial \tau^{2}}+|V|^{2} V \\
& =i \theta V+i \beta \frac{\partial^{2} V}{\partial \tau^{2}}-\frac{i \rho V}{1+|V|^{2} / P_{\mathrm{sat}}^{\mathrm{aver}}},
\end{aligned}
$$

where $V$ is the normalized envelope of the pulse, $Z=$ $z / L_{\mathrm{D}}$ is the normalized averaged propagation coordinate, $L_{\mathrm{D}}=T_{0}^{2} /\left|\beta_{2}\right|$ is the dispersion length, $\tau=t / T_{0}$ is the time normalized by the pulse duration $T_{0}$ and the other parameters are defined as

$$
\begin{aligned}
& D=-\operatorname{sgn}\left(\beta_{2}\right), \\
& \beta=\frac{g_{0} T_{2}^{2}}{2}\left|\beta_{2}\right|, \\
& \rho=\frac{L_{\mathrm{D}} \delta_{0} \Delta z}{2 L_{\mathrm{f}}}, \\
& \theta=L_{\mathrm{D}} \frac{g_{0}-k / L_{\mathrm{f}}}{2}, \\
& P_{\text {sat }}^{\text {aver }}=P_{\mathrm{sat}} L_{\mathrm{D}} \gamma \frac{1-\exp \left(-g_{0} L_{\mathrm{f}}\right)}{g_{0} L_{\mathrm{f}}}, \\
& V=U\left(L_{\mathrm{D}} \gamma \frac{\exp \left(g_{0} L_{\mathrm{f}}\right)-1}{g_{0} L_{\mathrm{f}}}\right)^{1 / 2},
\end{aligned}
$$

where $L_{\mathrm{f}}$ is the fiber length, which is assumed to be equal to the cavity length and $k$ is the output loss. A further simplification can be achieved by a Taylor expansion of the last term of Eq. (4) up to second order in $|V|^{2} / P_{\text {sat }}^{\text {aver }}$. Ultimately we obtain the established CQGLE given by

$$
\begin{aligned}
i \frac{\partial V}{\partial Z}+\frac{D}{2} & \frac{\partial^{2} V}{\partial \tau^{2}}+|V|^{2} V \\
& =i \delta V+i \beta \frac{\partial^{2}}{\partial \tau^{2}}+i \varepsilon|V|^{2} V-i \mu|V|^{4} V
\end{aligned}
$$

where the linear and nonlinear gain/loss coefficients are defined as

$$
\delta=\theta-\rho, \varepsilon=\frac{\rho}{P_{\mathrm{sat}}^{\mathrm{aver}}}, \mu=\frac{\rho}{\left(P_{\mathrm{sat}}^{\mathrm{aver}}\right)^{2}} .
$$

Hence, we can unambiguously relate the coefficients of the CQGLE and MGLE to the coefficients of the lumped model which reflect the experimental laser parameters.

\section{THE CQGLE MODEL}

\section{A. Bound state solutions}

To get a general picture of the two-SP interactions in the fiber laser we start our considerations from the distributed laser model, CQGLE (7). The reason is twofold, first most previous papers rely on this model and second, it is more appropriate for identifying the stationary solutions and applying the linear stability analysis.

From previous investigations 13, 14, 15, 16, 17, 18, $19,20,21$ ] it is known that BS formation is caused by linear and nonlinear dissipative effects (right hand side of Eq. (7)). Thus to simplify the analysis the number of system parameters was reduced from four $(\delta, \beta, \varepsilon, \mu)$ to two $(\beta, k)$ such as [16]:

$$
\delta=-k \beta / 3, \varepsilon=\beta / 2+k \beta, \mu=(5 / 8) k \beta,
$$

where the parameter $k$ characterizes the deviation from the particular point $(k=0)$ where arbitrary-amplitude solutions exist 31.

We are working in the normal dispersion regime $D=$ -1 with relatively strong spectral filtering $\beta=1$. To be sufficiently far from the singularity we choose $k=0.5$ where stable SPs exist. As initial conditions we use an in-phase superposition of two resting pulses separated by a certain distance. In the framework of the CGQLE the initial pulse profile is a critical issue with respect to BS formation. Thus for a better convergence we use as input two SPs, which are numerically obtained stationary onesoliton solutions for the respective parameter set [17].

To get a more complete picture of two-pulse solutions we consider the evolution for a wider range of initial separations and longer propagation distance than in previous works, see Fig. 1. We observe stationary BS formation beyond a certain distance (similar to [17]) and its succeeding destabilization, i.e. fusion of the pulses (below we discuss this instability in more detail). For an initial separation exceeding a critical value the SPs repel each other, was also observed in [16, 17]. However, a more careful study discloses that this separation is no ordinary repulsion but the formation of another BS with a larger peak separation (see Fig. 1). In previous works it was usually assumed that the interaction becomes too weak for such large peak separations and, hence, this scenario was not thoroughly investigated. The evolution to the second BS level has a much slower dynamics than that for the first one and needs an approximately 50 times longer propagation distance to form the stationary solution. This difference arises from the weaker SP interaction. Except for the larger formation distance and the slower formation dynamics there is no principal difference between these two BS levels. Similarly, beyond another critical initial separation we have observed the evolution towards a third BS level. Unfortunately, these calculations are too time-consuming, but intuitively it is clear that even higher order BS levels should occur. Evidently, it may be anticipated that the dynamics becomes progressively slower for higher levels and that all of them are unstable.

To double-check these expectations and to identify arbitrary BS levels, we solve the stationary problem using the relaxation and Newton's methods in combination. Figure 1 (right) shows results obtained from both the 

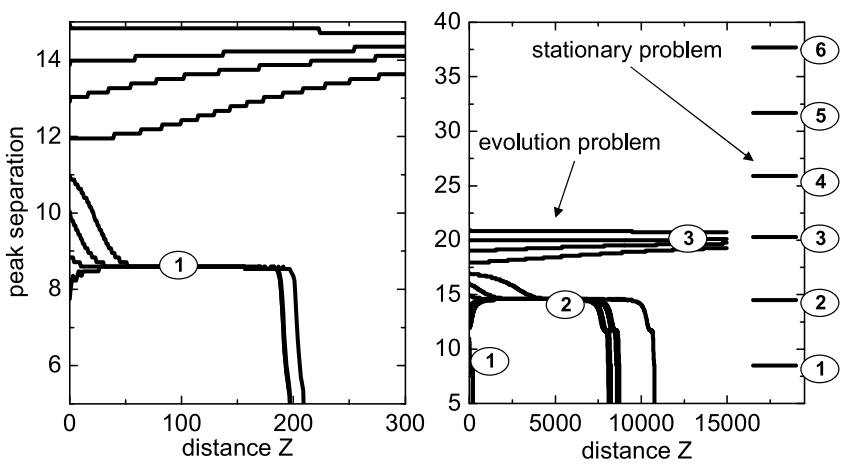

FIG. 1: Evolution of the separation for initially in-phase pulses with different initial separation. The levels of stationary in-phase BS solutions are displayed on the right axis on the right side. The peak separation is given in units of $\tau$; model: CQGLE (7); parameters: $k=0.5, \beta=1$.

propagation and from the stationary analysis, which are in perfect agreement in the intermediate section where the propagation is stationary. The solution of the stationary problem yielded six levels of in-phase BSs with different peak separation. It is interesting to see that the peak separation $S$ changes from level to level by a constant value (approximately $\Delta S=6$ for the current set of parameters) and obeys the simple equation $S_{\text {in-phase }}^{n} \approx 2.5+6 n$ where $n=1,2,3, \ldots$ designates the BS level.

Also the first six levels of the out-of-phase stationary BS solutions were found. Similarly, the dependence of the peak separation on the level (except the first one) can be approximated by $S_{\text {out-of-phase }}^{n} \approx 5.5+6 n$, where $n=2,3,4, \ldots$, while for the first level $S_{\text {out-of-phase }}^{1}=4.7$. We guess that this peculiarity of the first level is caused by the strong SP overlap resulting in enhanced nonlinear effects.

We note that for a $\pi / 2$ phase difference between the pulses neither stable nor unstable BS solutions have been found in the normal dispersion regime.

To understand the multilevel nature we consider the BS intensity and phase profiles for the first three levels, shown in Fig. 2. From this figure we may draw two conclusions. First, the intensity profile of in-phase (out-ofphase) BS looks always the same, independently of the level, and has a characteristic small dip in the center. Second, there is a definite phase relation between the BS constituents and between the levels. For example, for inphase BS levels the phase difference between the phase maximum and the phase minimum (at the center) is a multiple of $\pi$ and can be written as $\Delta \varphi_{\mathrm{in} \text {-phase }}^{n} \approx \pi n$, where $n=1,2,3, \ldots$ is the BS level. For out-of-phase solutions this phase difference can be approximated by $\Delta \varphi_{\text {out-of-phase }}^{n} \approx 0.8+\pi n$, where $n=2,3,4, \ldots$, while for the first level $\Delta \varphi_{\text {out }- \text { of }- \text { phase }}^{1}=3.6$.

Physically, the equidistant multilevel nature of the BSs can be understood as constructive interference between SPs and can be clearly seen from the almost linearly

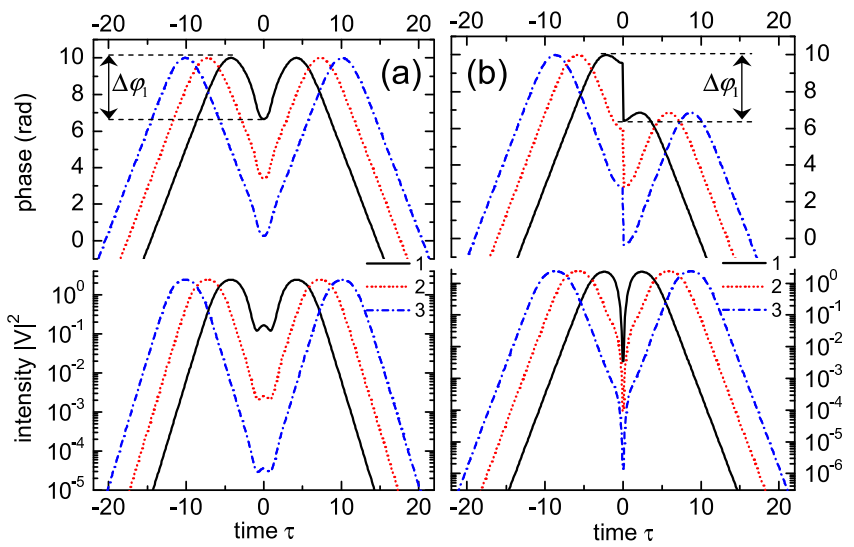

FIG. 2: (Color online) Intensity and phase profiles for the first three levels of the (a) in-phase and (b) out-of-phase stationary BS solutions; model: CQGLE (7); parameters: $k=0.5, \beta=$ 1 .

growing phase of the tails (Fig. 2). On the other side, our results are in qualitative agreement with Malomed's analysis [14, 18] where the oscillating tails of the SPs (linear phase change) evoke a periodic interaction potential against the pulse separation, in spite of the fact that this analysis [14, 18] is based on a NLSE perturbation approach and is valid just for the anomalous dispersion regime. Moreover, we propose the magnitude $\Delta \varphi$ as a convenient parameter for the definition of the BS level in simulations or experiments.

We visualize the evolution of both pulses in a phase plane $(\rho(z), \phi(z))$ where $\rho(z)$ is the peak separation and $\phi(z)$ their relative phase (difference of the phase between peaks), see Fig. 3. In this plot we analyze the evolution trajectories of two pulses for two relevant cases. In the first case we consider two out-of-phase SPs with an initial separation of $S=7$ which are evolving towards the first level out-of-phase stationary BS solution. In the second case the evolution of a pair of in-phase SPs with a larger initial separation of $S=16.1$ (evolving towards the second level in-phase stationary BS solution) is displayed. The evolution trajectory for the first case, which may be partly described by a circle indicated by '1' in Fig. 3, is well studied [17]. The radius of this circle equals the distance between the peaks of the first level out-of-phase BS.

To date, it was not shown that in the second case, where the initial separation between the solitons is 16.1, the evolution trajectory partly consists of four circles. From the plot (Fig. 3) we can recognize that each circle corresponds to a certain BS. The radius of the biggest one equals the distance between pulses of the second level in-phase BS, being in agreement with the previous case. The other circles ' 3 ', '2' and '1' correspond to the second level out-of-phase, first level in-phase and first level outof-phase stationary BS solutions, respectively.

It is interesting to note that the evolution trajectory of two in-phase or out-of-phase SPs attains simple geo- 


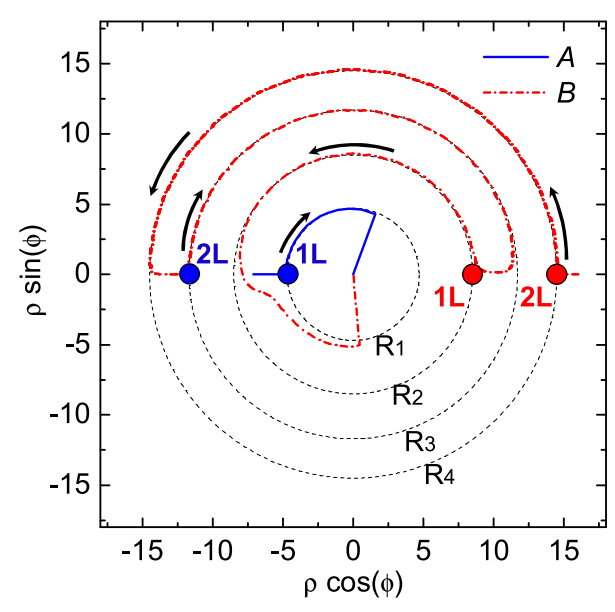

FIG. 3: (Color online) Soliton trajectories in the phase plane for: two initially out-of-phase solitons with separation 7 (line $A)$ and two initially in-phase solitons with separation 16.1 (line $B$ ). 1L - first level stationary in-phase (red right) and out-of-phase (blue left) bound state solution, 2L - second level stationary in-phase (red right) and out-of-phase (blue left) bound state solution; model: CQGLE (7); parameters: $k=$ $0.5, \beta=1$.

metrical forms. Moreover, during the evolution they pass through all possible in-phase and out-of-phase stationary BS solutions with ever smaller peak separation.

This can be explained by the earlier finding that the phase difference between the pulses depends on their separation as a consequence of interference phenomena between them.

\section{B. Linear stability analysis}

In previous papers [17, 18, 21] it was shown that inphase and out-of-phase first level BS are unstable. From our propagation simulations we anticipate that the corresponding higher level BSs are unstable as well. In order to confirm this hypothesis and to provide a more complete picture we carry out a linear stability analysis of the higher BS levels. We start with the first level in-phase stationary solution. Its formation, the typical instability behavior and final fusion of the two-pulse excitation are shown in Fig. 4. It is interesting to note that the instability exhibits a temporal asymmetry in the absolutely symmetric CQGLE.

To perform the linear stability analysis we perturb the stationary solution $V_{0}(t) \exp \left(i k_{0} z\right)$ as $V(z, t)=V_{0}(t) \exp \left(i k_{0} z\right)+\delta V(z, t)=$ $\left[V_{0}(t)+f \exp (\lambda z)+g \exp \left(\lambda^{*} z\right)\right] \exp \left(i k_{0} z\right)$, where $V_{0}(t)$ was numerically calculated and $f, g$ are the small amplitudes of the perturbation. The substitution of this ansatz into the CQGLE (7) and its respective linearization in $f, g$ leads to a system of linear, homogeneous equations. The solvability condition provides the eigenvalues $\lambda$. Eigenvalues with positive real part

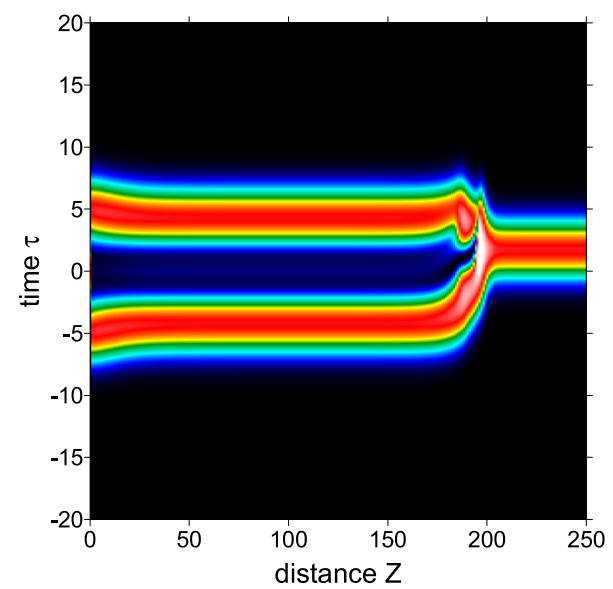

FIG. 4: (Color online) Bound state instability. Two inphase solitons form a quasi-stationary bound state but fuse asymmetrically eventually; model: CQGLE (7); parameters: $k=0.5, \beta=1$.
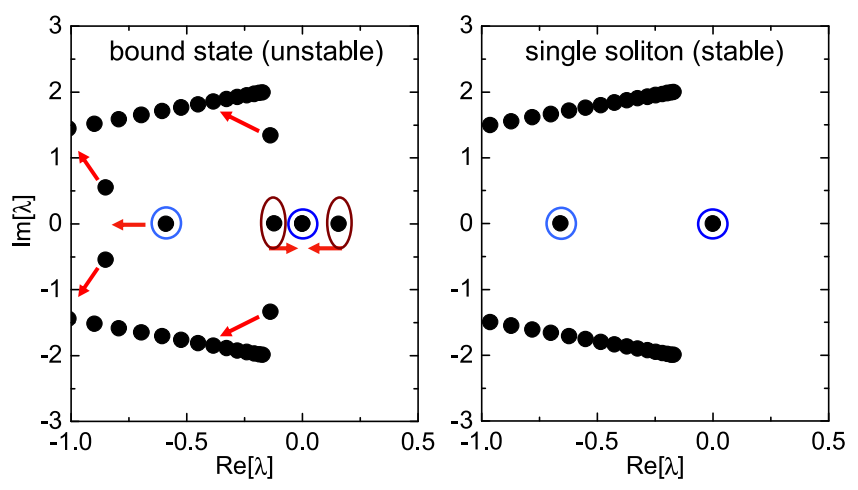

FIG. 5: (Color online) Complex eigenvalues for both the first level unstable stationary in-phase BS and stable SP solution. The arrows denote the directions where the eigenvalues are shifted for higher levels BSs with decreasing interaction; model: CQGLE (7); parameters: $k=0.5, \beta=1$.

indicate that the perturbation grows upon propagation and the stationary solution destabilizes. In Fig. 5 the calculated eigenvalues for the unstable first level in-phase BS are displayed and compared with the eigenvalues of the stable SP solution.

Evidently, in the limit of two infinitely separated SPs the eigenvalues coincide with those of the stable single $\mathrm{SP}$ solution. In this case the zero eigenvalue exhibits a fourth order degeneracy corresponding to the two phase and two translation modes. If the peak separation decreases the pulses start to perturb each other affecting the eigenvalues. Because of interaction two eigenvalues branch off from the zero eigenvalue and move in opposite directions on the real axis for decreasing separation. Thus, the eigenvalue with the positive real part is responsible for the instability of the BS solution, see Fig. 5. The two eigenmodes corresponding to these eigenvalues are shown in Fig. 6. They are formed as linear combinations 


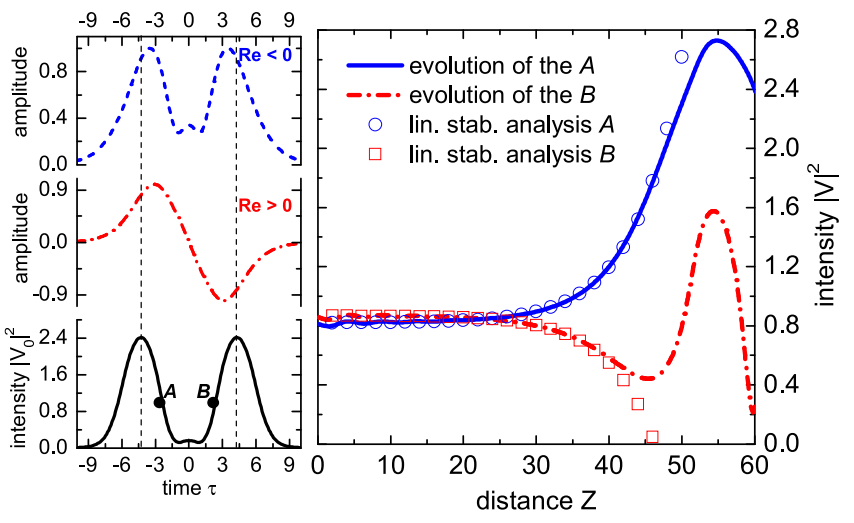

FIG. 6: (Color online) Left: Perturbation modes (dotted blue line and dashed red line) of the first level in-phase BS (solid black line). Right: Evolution trajectories of points $A$ and $B$ of the stationary BS solution. The amplitude of the perturbation modes is given in arbitrary units; model: CQGLE (7); parameters: $k=0.5, \beta=1$.

of the four individual eigenmodes of the original SP (two phase modes, two translation modes) shifted by the distance $S$. The peaks of both modes are shifted towards the center and do not coincide with the peaks of the BS. Also because of SPs interaction another four eigenvalues are symmetrically split off the spectral continuum and move away from it for decreasing BS level. However, all of them have a negative real part and do not affect the stability of the bound state solution.

From the results presented in Figs. 5 and 6 we may conclude that the antisymmetric mode is responsible for the BS instability. In the propagation simulations this mode is excited by noise and then grows with $\operatorname{Re}(\lambda)>0$. To confirm the results of the linear stability analysis we add white noise to the stationary BS solution and plot the evolution trajectories of two points (fixed on the time coordinate, calculated in the propagation scheme). The points are chosen to be close to the amplitude maxima of the unstable mode, see Fig. 6 (left). The growing antisymmetric eigenmode evokes an increase of the amplitude in one point and a decay in the other point upon propagation. Figure 6 (right) shows that the results of the linear stability analysis and the exact numerical propagation simulations perfectly coincide up to the distance where the linear stability analysis ceases to be valid.

As expected, $\operatorname{Re}(\lambda)$ calculated for other BS levels is smaller for higher levels, see Table I This dependency correlates well with the slower dynamics (evolution to the BS solution and instability dynamics) discussed in Fig. 1. From a mathematical and practical point of view the growth rate $\operatorname{Re}(\lambda)$ for higher BS levels can be very close to zero, but physically it remains an unstable BS.

We varied the CQGLE parameters $\beta, k$ and $\delta$ to find in-phase or out-of-phase stable BSs but failed.
TABLE I: $\operatorname{Re}(\lambda)$ for different levels of stationary BS solutions; model: CQGLE (7); parameters: $k=0.5, \beta=1$.

\begin{tabular}{c|c|c|c|c} 
& \multicolumn{2}{|c|}{ in-phase } & \multicolumn{2}{c}{ out-of-phase } \\
level & $\begin{array}{c}\text { peak } \\
\text { separation }\end{array}$ & $\operatorname{Re}(\lambda)$ & $\begin{array}{c}\text { peak } \\
\text { separation }\end{array}$ & $\operatorname{Re}(\lambda)$ \\
\hline 1 & 8.5 & $1.57 \cdot 10^{-1}$ & 4.7 & $5.95 \cdot 10^{-1}$ \\
2 & 14.5 & $4.21 \cdot 10^{-3}$ & 11.7 & $3.11 \cdot 10^{-2}$ \\
3 & 20.3 & $6.09 \cdot 10^{-5}$ & 17.3 & $5.11 \cdot 10^{-4}$ \\
4 & 25.9 & $8.60 \cdot 10^{-7}$ & 23.1 & $7.23 \cdot 10^{-6}$ \\
5 & 31.7 & $1.17 \cdot 10^{-8}$ & 28.7 & $1.02 \cdot 10^{-7}$ \\
6 & 37.7 & $1.13 \cdot 10^{-10}$ & 34.7 & $6.10 \cdot 10^{-10}$
\end{tabular}

\section{THE LUMPED MODEL}

In this section we study BS formation in fiber ring lasers using the more realistic lumped model and experimentally accessible laser parameters. The aim of this study is manifold. First of all, this model reflects better the experimental situation. So, it is interesting to compare the results with those of the distributed model which is easier to handle but contains severe approximations. Moreover, we will study the effect of the finite SA response time (noninstantaneous model) on the BS stability. A stabilization has been proven in the spatial domain by taking advantage of nonlocality (see 32] and references therein). Here, the noninstantaneous response plays the role of nonlocality in the spatial domain. Eventually we will summarize the differences between the results provided by the lumped and the distributed model in order to derive the limits of validity of the latter.

For the simulation we use parameters closely related to previous experiments at a carrier wavelength of $1030 \mathrm{~nm}$ 22, 23, 27]. The length of the absorber and the smallsignal loss were adjusted to a modulation depth of $30 \%$, the relaxation time of the fast, but noninstantaneous, SA is $500 \mathrm{fs}$, its saturation energy amounts to $16.7 \mathrm{pJ}$ and the respective saturation power to $P_{\text {sat }}=33.4 \mathrm{~W}$. The output loss is equal to $30 \%$. For the doped fiber we assume $L_{\mathrm{f}}=1 \mathrm{~m}, \beta_{2}=0.024 \mathrm{ps}^{2} \mathrm{~m}^{-1}, \gamma=0.005 \mathrm{~W}^{-1} \mathrm{~m}^{-1}$ and $E_{\mathrm{sat}}^{\mathrm{Gain}}=1 \mathrm{~nJ}$. Amplitude and phase profiles were always recorded after the output coupler.

In order to trigger BS formation we varied the filter strength, corresponding to the inverse gain bandwidth $T_{1}$ of the laser material, and the small-signal gain.

\section{A. Instantaneous SA approximation}

In a first step we aim at reproducing the BS levels obtained in the CQGLE model. For this purpose we start with the instantaneous SA approximation, Eq. (4). As initial condition we use two in-phase or out-of-phase resting small amplitude Gaussian pulses which converge to the BS. Because of the additional stabilization effect of the saturated gain the current model is less critical with respect to the initial pulse profiles than the CQGLE. We observe BS formation for $T_{1}=300 \mathrm{fs}$ and $g_{0}=0.75 \mathrm{~m}^{-1}$. 


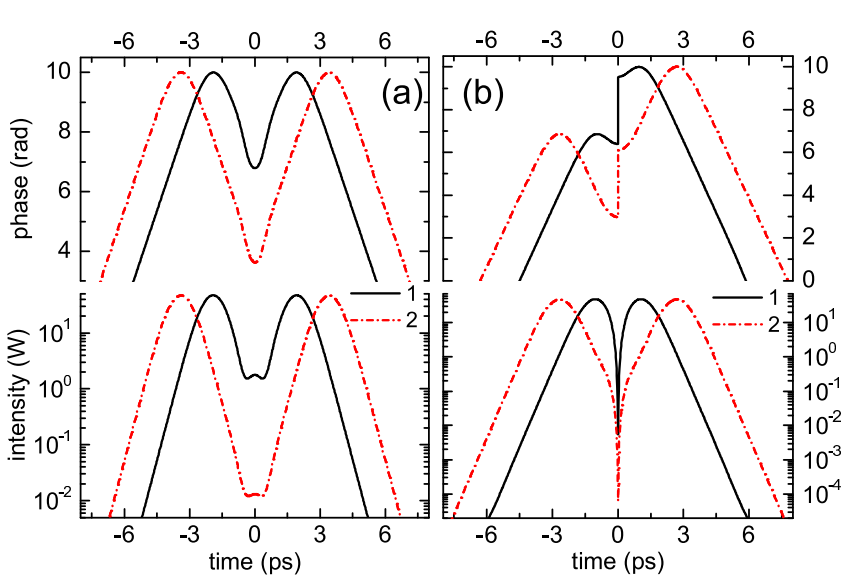

FIG. 7: (Color online) Intensity and phase profiles for first (solid line 1) and second (dashed line 2) levels of the stationary BS solutions; (a) - in-phase, (b) - out-of-phase; lumped model (parameters, see text), instantaneous SA approximation (4).

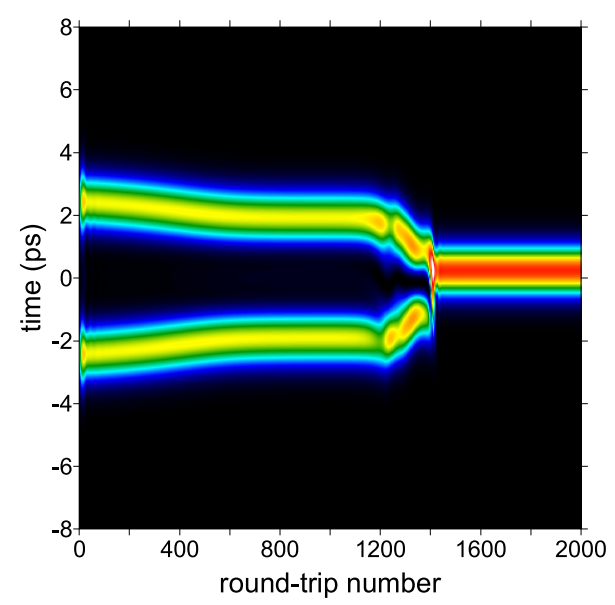

FIG. 8: (Color online) Bound state instability. The two inphase pulses form the stationary bound state and fuse asymmetrically eventually; lumped model (parameters, see text), instantaneous SA approximation (4).

The first and second stationary in-phase BS levels are displayed in Fig. 7. In-phase BSs exhibit a small characteristic central peak in the intensity profile and the difference between phase maximum and minimum (at the center) is a multiple of $\sim \pi$. In agreement with our previous results the formation of the second level in-phase BS needs approximately 80 times more $(40,000)$ round trips than the 1st level (500). However, also these stationary BS solutions are unstable with an asymmetric instability behavior which is similar to the CQGLE BSs, see Fig. 8.

For out-of-phase initial conditions we have also observed first and second level stationary out-of-phase BSs, see Fig. 7. They exhibit a similar formation dynamics and instability behavior as in-phase solutions. In particular, the formation of a level 2 out-of-phase BS needs approximately 30 times more $(3,000)$ round-trips than that for a level 1 out-of-phase BS (100).

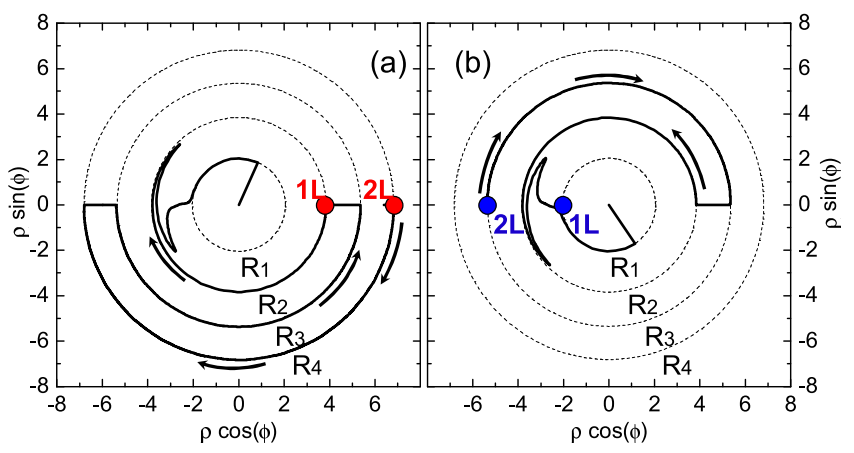

FIG. 9: (Color online) Pulse trajectories in the phase plane for two pulses (a) initially in-phase with separation 6.38 ps and (b) initially out-of-phase with separation 5.25 ps. 1L - first level BS, 2L - second level BS; lumped model (parameters, see text), instantaneous SA approximation (4).

To visualize the interactions between both pulses we again take advantage of the phase plane. We consider the evolution trajectories for initially in-phase and outof-phase pulses, see Fig. 9. For in-phase pulses the trajectory is partly described by four circles. As we already know, each circle $\left(R_{1}, R_{2}, R_{3}, R_{4}\right)$ corresponds to a certain level of out-of-phase or in-phase stationary BS solutions, shown in Fig. 9. There is essentially no difference between the evolution trajectories provided by the lumped and the distributed model.

\section{B. Differences between models}

For the sake of comparison between the different models and of evaluating their validity we use the instantaneous SA approximation and compare results provided by the lumped model, which serves as a benchmark, with both distributed models, the MGLE (5), which accounts for the full SA saturation, and the CQGLE (7), which relies on the Taylor expansion of the SA saturation.

Figure 10 shows stable SP solutions obtained by both the lumped and the MGLE model. For the lumped modeling the saturation energy of the gain was reduced by a factor of two, because we consider just a single pulse. The MGLE parameters were calculated from those of the lumped model, except that for the distributed model a proper magnitude of the small-signal gain (6) was adjusted as $g_{0}=0.6388 \mathrm{~m}^{-1}$, corresponding to the gain in the lumped model for stationary conditions. It is interesting to note that for the relevant parameter set stable SP solutions exist in the MGLE, but not in the CQGLE model. This is a clear indication that the saturation behavior plays a pivotal role. Usually mode-locked lasers are working in a regime where the absorber is saturated, i.e., $|V|^{2} / P_{\text {sat }}^{\text {aver }}<1$ is not satisfied and the CQGLE is incorrect. Hence, one must not expect solutions for similar parameters in the CQGLE model on the one side and the lumped (or MGLE) model on the other side. Nevertheless, the MGLE and the lumped model with an ideal 

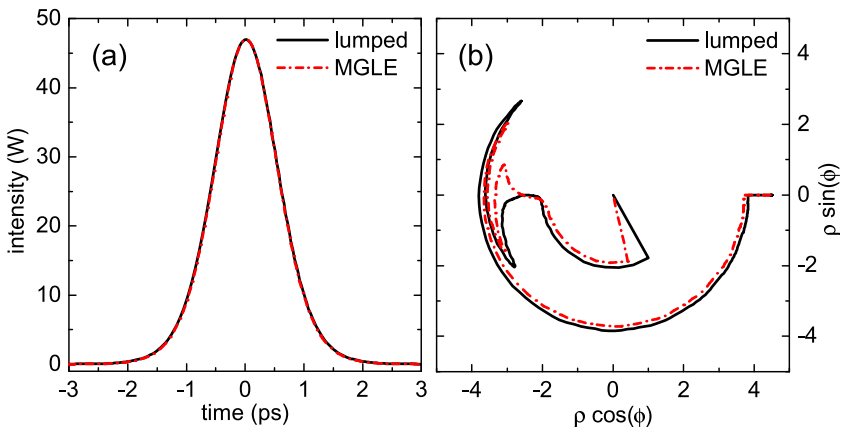

FIG. 10: (Color online) Comparison between the lumped and the MGLE model at the same parameters set. (a) - intensity profile of stable SP solutions, (b) - evolution trajectories of two pulses in the phase plane; instantaneous SA approximation (4).

SA show quite a good agreement regarding the stable SP solutions, see Fig. 10.

Further on we consider the evolution of two SPs in the phase plane, see Fig. 10 (in this case we use the original value of the saturation energy $E_{\mathrm{sat}}^{\mathrm{Gain}}=1 \mathrm{~nJ}$ ). Although minor differences occur the evolution trajectories of the two pulses behave quite similar for both the MGLE and the lumped model.

\section{Noninstantaneous SA response}

In a next step, relying on the lumped model, we are attempting to achieve BS stabilization by accounting for the noninstantaneous SA response (3). This model reflects the experimental situation because typical SA response times vary between 300 fs and 12 ps, which frequently compares to the pulse length. We use the parameters from the previous section $\mathbf{A}$, in order to keep the saturation power constant, and account for the variation of the relaxation time by a proper change of the saturation energy $E_{\mathrm{sat}}^{\mathrm{SA}}$.

In these simulations as initial condition we use two resting pulses with small amplitudes. At the beginning the pulses propagate 100 round trips in the ideal SA approximation and just then we switch on the noninstantaneous response. This procedure is required to arrive at a definite initial condition for large amplitude bound states.

Trivially, for a vanishing relaxation time the system exhibits unstable stationary BS solutions identical to them of the instantaneous SA approximation. With increasing relaxation time (about $150 \mathrm{fs}$ ) we observe a tendency towards BS stabilization with moderate oscillations. Eventually the BSs stabilize for a relaxation time (about 350 fs) close to the experimental situation, see Figs. 11, 12. With a further increase of the relaxation time the arising stable BSs exhibit a fixed peak separation. The solutions move backwards in the reference frame due to the temporal effects in the absorber, see Fig. 12.

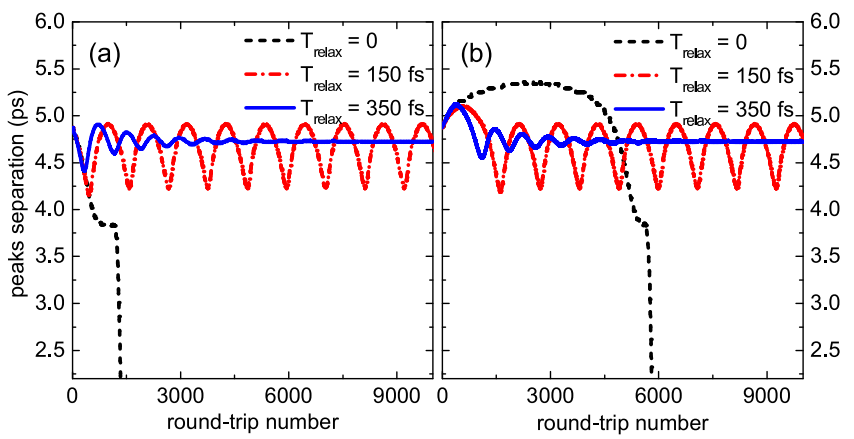

FIG. 11: (Color online) Bound state stabilization. Distance between two pulses as a function of round-trips for initially (a) - in-phase and (b) - out-of-phase pulses for different SA relaxation times. In the limit $T_{\text {relax }}=0$, pulses build (a) the level 1 in-phase stationary BS solution, (b) the level 2 out-ofphase stationary BS solution; lumped model (parameters, see text), noninstantaneous SA response (3).

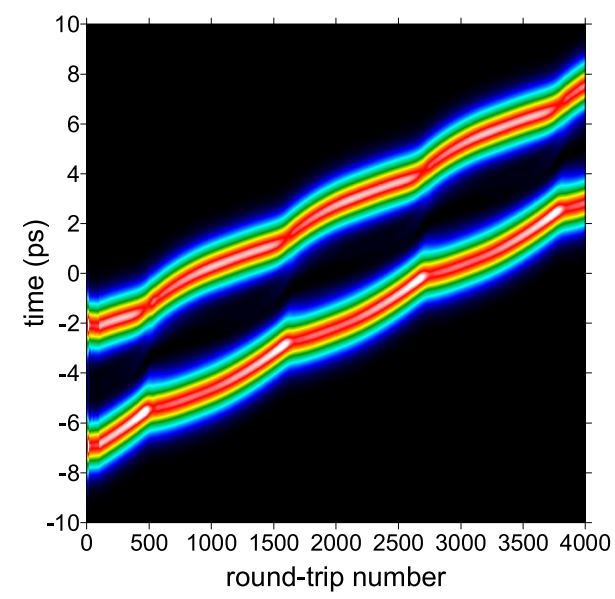

FIG. 12: (Color online) Oscillating BS solution for $T_{\text {relax }}=$ 150 fs; lumped model (parameters, see text), noninstantaneous $\mathrm{SA}$ response (3).

It is interesting to note that the resulting stationary and oscillating stable BS solutions do not depend on the initial phase difference between the pulses, see Fig. 11. This is in stark contrast to the CQGLE model, where the initial phase difference defines the nature of the stationary BS solution. There, two in-phase or out-of-phase pulses with identical separation evolve towards different levels (see the curve for $T_{\text {relax }}=0$ in Fig. 11). Moreover, we have observed stationary and oscillating stable BS solutions of the next level with a larger peak separation. To obtain them the initial distance between pulses was increased and proper figures for the relaxation time were chosen. Higher level BSs need approximately 100 times more round-trips for their formation, which is in agreement with previous results.

The nature of stationary and oscillating stable BS solutions can be understood by inspecting the trajectories in the phase plane, see Fig. 13. We evaluate the two 


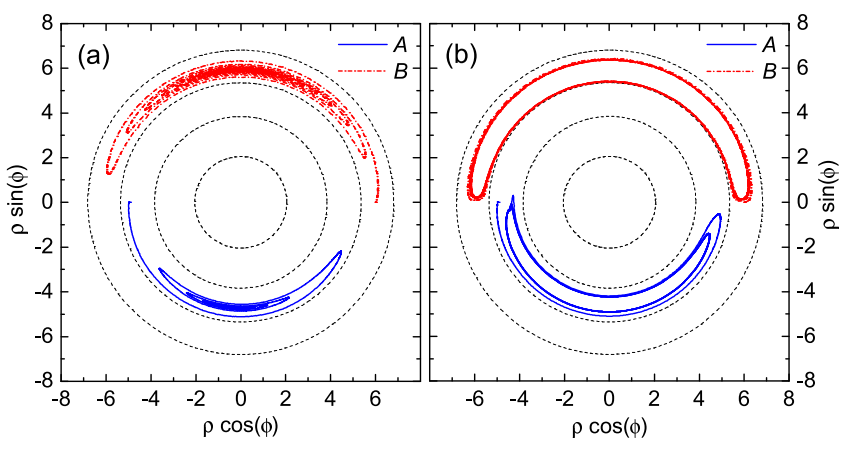

FIG. 13: (Color online) Trajectories showing the two-pulse evolution; a) 1st level stable BS solution $T_{\text {relax }}=350 \mathrm{fs}$ (blue line $A), 2$ nd level stable BS solution $T_{\text {relax }}=75 \mathrm{fs}($ red line $B)$ and (b): 1st level oscillating BS solution $T_{\text {relax }}=150 \mathrm{fs}$ (blue line $A$ ), 2nd level oscillating BS solution $T_{\text {relax }}=5 \mathrm{fs}$ (red line $B)$; lumped model (parameters, see text), noninstantaneous SA response (3).

pulse dynamics in phase space for four different cases. To generate the first and second level BSs we use two pulses with an initial separation of $4.9 \mathrm{ps}$ and $6 \mathrm{ps}$, respectively. Moreover, for each case we adjust the proper relaxation time in order to obtain a stationary or an oscillating stable BS solution. Figure 13 shows that stationary BS solutions are fixed points in the phase plane which are located near $\phi=\pi / 2$. Oscillating solutions move either in the lower or upper half plane between two adjacent circles.

The conclusion to be drawn is that stationary stable BS solutions (Fig. 14) of the lumped model with noninstantaneous SA are neither in- nor out-of-phase but exhibit a phase difference of about $\pi / 2$ between the pulses. Immediately the question arises whether there are stable BS solutions with $\pi / 2$ phase difference between the pulses in the instantaneous models (CQGLE or lumped model with instantaneous SA). In the lumped model no stable or even stationary BS solutions with that phase difference could be identified. For the CQGLE model, but just for anomalous dispersion, this case has been studied earlier [21], and it was shown, that stable BS solutions can exist.

Nevertheless, stable BS solutions in the lumped model with noninstananeous SA differ considerably from those discussed in 21]. First, they exist for a completely different set of parameters (even opposite dispersion) and second, their behavior in phase space (Fig. 13) exhibits a large difference to those studied in 21]. Because all SAs have a finite relaxation time the conclusion can be drawn that the parameter range for the existence of BSs is much wider than assumed in [21].

As already mentioned BS stabilization is evoked by the noninstantaneous SA response in analogy to the spatial case where a nonlocality stabilizes localized solutions [32]. Here, the retarded temporal SA response is asymmetric in time, leading to the minor deviation of the fixed point from $\phi=\pi / 2$. By contrast, the stable BS solutions of

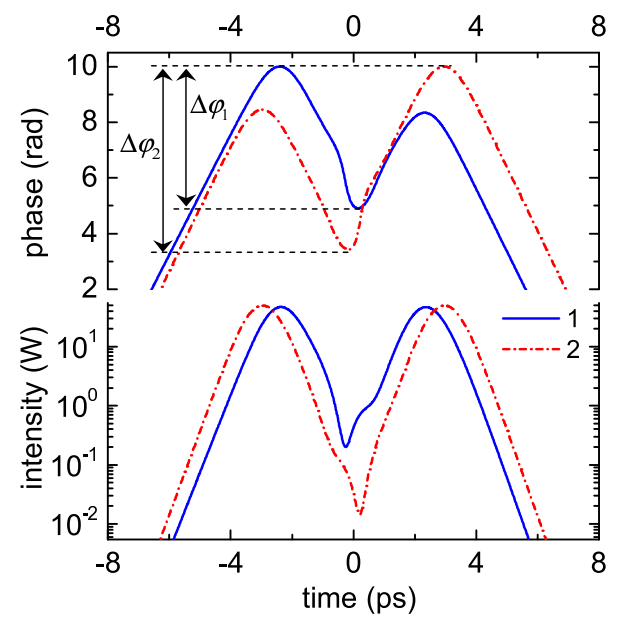

FIG. 14: (Color online) The intensity and phase profiles for 1st and 2nd level stable BS solutions calculated for $T_{\text {relax }}=$ $350 \mathrm{fs}$ (blue solid line 1), $T_{\text {relax }}=75 \mathrm{fs}$ (red dashed line 2). The phase differences are $\Delta \varphi_{1}=5.1$ and $\Delta \varphi_{2}=6.6$; lumped model (parameters, see text), noninstantaneous SA response (3).

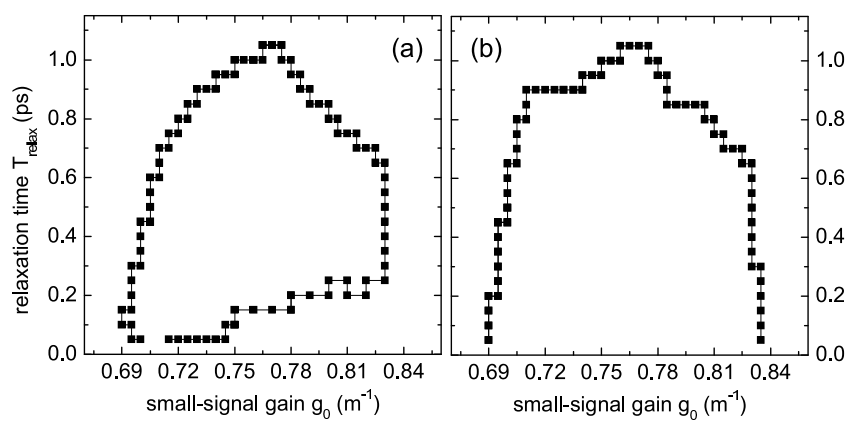

FIG. 15: Existence domains for stable (stationary and oscillating) bound state solutions; (a) first level solution, (b) second level solution; lumped model (parameters, see text), noninstantaneous SA response (3).

the CQGLE appear exactly for $\phi=\pi / 2[21]$.

The region of existence of stable BSs is displayed in Fig. 15. It can be recognized that the regions for two neighboring BS levels differ, especially the bottom boundaries. This means that oscillating solutions of the first and second level can be observed for different relaxation times and the same small-signal gain. The open boundary indicates that the bottom margin is very close to the zero axis.

A distinctive feature of stable BS solutions is their behavior in the phase plane. The two initially resting pulses, for a certain range of initial separation, always evolve towards a single fixed point independently of the initial phase difference. This is in stark contrast to the stable BS solutions studied in Ref. 21] where two similar solutions are possible, lying symmetrically on opposite sides of the phase plane. Moreover, the neighboring levels of BSs are situated in different half spaces, see Fig. 
13. This is due to the lack of invariance of the time scale if relaxation matters. Thus leading and trailing pulses are not equivalent.

Figure 13 shows that each fixed point on the phase plane lies between two neighboring circles. Thus one might anticipate that there is a fixed point between circle 1 and 2 too. But in our simulations we did not succeed in finding any stable BS in that domain.

\section{CONCLUSION}

In conclusion, we have theoretically and numerically investigated the formation and stability of BSs in a modelocked fiber ring laser with SA in the normal dispersion regime. We started our investigations with the distributed description of the laser which is given by the commonly used CQGLE. For the first time, to the best of our knowledge, we have observed a discrete family of stationary BS solutions with different peak separation. Moreover, the observed BS levels exhibit an equidistantly increasing peak separation with a well-defined phase relation. All levels of in-phase and out-of-phase BSs turned out to be unstable for the investigated system parameters. A linear stability analysis has shown that this instability is caused by the antisymmetric perturbation mode. We found that both the formation dynamics of BSs and their succeeding decay is slower for higher levels because of the weaker interaction. The evolution trajectories in the phase plane of two SPs with relatively large initial separation consist of simple geometrical forms.

All these findings could be confirmed by using a more realistic lumped laser model but maintaining the instantaneous SA response.

Stabilization of the BSs was achieved in the lumped laser model by taking the fast, nevertheless noninstantaneous SA response into account. Using the SA relaxation time as control parameter a continuous transition from unstable to stable BS solutions may be achieved passing a domain of oscillating BS solutions. These stationary and oscillating stable BSs have a discrete multilevel nature too. The distinctive feature of these BS solutions is expressed in the phase plane. A stable BS solution of a certain level represents a fixed point in the phase plane, which lies in the prefered half space. For oscillating BSs the evolution is described by circulations between two neighboring circles in either the lower or upper half space depending on the level. The stabilization mechanism identified shows that all lasers with semiconductor absorber have a wider region of stable BS solutions than previously assumed [21]. Moreover, we have shown that a discrete family of stable or oscillating BS solutions with equidistant peak separation can be obtained in such laser systems.

The results presented can be used in optics communication lines for generating multipulse trains with well defined separation distances or in fiber lasers for an irregular or regular pulse array generation.

\section{Acknowledgments}

This work was supported by the Deutsche Forschungsgemeinschaft (research unit 532). Rumen Iliew acknowledges financial support from the Carl Zeiss foundation.
[1] L. F. Mollenauer, R. H. Stolen, J. P. Gordon, Phys. Rev. Lett. 45, 1095 (1980)

[2] V. I. Karpman and V.V. Solov'ev, Physica D 3, 487 (1981)

[3] J. P. Gordon, Opt. Lett. 8, 596 (1983)

[4] D. Anderson and M. Lisak, Opt. Lett. 11, 174 (1986)

[5] V. V. Afanasjev and V. A. Vysloukh, J. Opt. Soc. Am. B 11, 2385 (1994)

[6] A. Hasegawa and F. Tappert, Appl. Phys. Lett. 23, 142 (1973)

[7] G. P. Agrawal, Nonlinear fiber optics, 2nd edition (Academic, London, 1995)

[8] J. P. Gordon, Opt. Lett. 11, 662 (1986)

[9] Y. Kodama and A. Hasegawa, IEEE J. Quantum Electronics 23, 510-524 (1987)

[10] A. Hasegawa and Y. Kodama, Proc. IEEE 69, 1145 (1981)

[11] N. N. Akhmediev, A. Ankiewicz, Solitons: Nonlinear Pulses and Beams (Chapman \& Hall, London, 1997)

[12] P.-A. Belanger, Opt. Express 13, 8089 (2005)

[13] H. R. Brand and R. J. Deissler, Phys. Rev. Lett. 63, 2801 (1989)

[14] B.A. Malomed, Phys. Rev. A 44, 6954 (1991)

[15] V. V. Afanasjev, Opt. Lett. 18, 790 (1993)
[16] V. V. Afanasjev and N. Akhmediev, Opt. Lett. 20, 1970 (1995)

[17] V. V. Afanasjev and N. Akhmediev, Phys. Rev. E 53, 6471 (1996)

[18] V. V. Afanasjev, B. A. Malomed, and P. L. Chu, Phys, Rev. E 56, 6020 (1997)

[19] I. M. Uzunov, R. Muschall, M. Glles, F. Lederer, S. Wabnitz, Opt. Commun. 118, 577 (1995)

[20] S. Wabnitz and E. Westin, Opt. Lett. 21, 1235 (1996)

[21] N. N. Akhmediev, A. Ankiewicz, J. M. Soto-Crespo, Phys, Rev. Lett. 79, 4047 (1997)

[22] B. Ortac, O. Schmidt, T. Schreiber, J. Limpert, A. Tünnerman, and A. Hideur, Opt. Express 15, 10725 (2007)

[23] A. Chong, W. Renniger, and F. Wise, Opt. Lett. 32, 2408 (2007)

[24] A. Chong, W. Renniger, and F. Wise, J. Opt. Soc. Am. B 25, 140 (2008)

[25] G. P. Agrawal, Phys. Rev. A 44, 7493 (1991)

[26] G. Agrawal, Applications of nonlinear fiber optics (Academic, 2001), chapters 4 and 5

[27] F. O. Ilday, J. R. Buckley, W. G. Clark, and F. W. Wise, Phys. Rev. Lett. 92, 213902 (2004)

[28] G. Agrawal and N. Olsson, IEEE J. Quantum Electron., 
QE-25, 2297 (1997)

[29] A. Hasegawa and Y. Kodama, Opt. Lett. 15, 1443 (1990)

[30] N. N. Akhmediev, A. Ankiewicz, Dissipative solitons (Springer, Berlin Heidelberg, 2005), chapter Dynamics of dissipative temporal solitons
[31] V. V. Afanasjev, Opt. Lett. 20, 704 (1995)

[32] S. Skupin, M. Grech, W. Krolikowski, Opt. Express 16, 9118 (2008) 